\title{
Twitter Analysis of Covid-19 Misinformation in Spain
}

\author{
Diego Saby ${ }^{1}$, Olivier Philippe ${ }^{1}$, Nataly Buslón ${ }^{1}$, Javier del Valle ${ }^{1}$, Oriol Puig ${ }^{1}$, \\ Ramón Salaverría ${ }^{2}$, and María José Rementeria ${ }^{1}$ \\ 1 Barcelona Supercomputing Center, Barcelona, Spain \\ ${ }^{2}$ Universidad de Navarra
}

\begin{abstract}
A graph analysis on the tweets and users networks from a set of curated news was done to study the existing difference in communication patterns between legitimate and misinformation news. Our findings suggest there is no difference in the influence of misinformation and legitimate news but misinformation news tend to be more shared and present than legitimate news, meaning that while misinformation tweets do not have more influence, their authors are more prolific. Misinformation reach wider audience even if the tweets, individually, are not more influential. A subsequent qualitative analysis on the users reveal that there is also influence of misinformation spreading in Spain from other Spanish speaking countries.
\end{abstract}

Keywords: Misinformation diffusion - Twitter influence $\cdot$ Network analysis.

\section{Introduction}

The diffusion of misinformation and disinformation through modern communication and social networking sites is one of today's most urgent problems. The situation has worsened in recent years since its circulation has reached unforeseen scales, severely affecting domains that range from politics and economy to public health. In healthcare, the proliferation of manipulated medical information has been perceived as especially harmful due to the impact that this content might have on people's lives [15].

We understand disinformation as verifiably false or misleading information created, presented and disseminated for economic gain or to intentionally deceive the public, while misinformation is verifiably false information that is spread without the intention to mislead ${ }^{3}$. With the rapid spread of misinformation in social networks during the COVID-19 pandemic and the extensive use of Twitter as information platform [8], a large number of studies have been carried out since then. For example, Singh et al. analyse the most prevalent myths about the COVID-19 and the number of conversations about those myths over time 14. Authors in 44 classify misinformation in some categories and try to find

\footnotetext{
3 https://digital-strategy.ec.europa.eu/en/policies/online-disinformation
} 
who is interacting with influential tweets containing misinformation and where those tweets are propagated in the global network. In [7, authors develop a twitter misinformation dataset and analyses the communities inside the users interactions network. Mcquillan et al. present a network community analysis and topic analysis about COVID-19 topics in Twitter [6].

As part of the RRSSalud project Dynamics of dissemination in social networks of false news about health, 2020-2022 24, we leveraged data from Twitter to study the dynamics of dissemination of health, politics and technology misinformation and disinformation.

Additionally, we examined the dynamics of tweets written in Spanish and their profiles in the context of the COVID-19 pandemic.

\section{Dataset and methodology}

Our study has been carried out with a dataset composed by misinformation and legitimate news. The subset was built during the RRSSalud project 13 selecting 10 misinformation news on health, technology and politics published between March and April 2020 5 We completed our dataset with 10 verified news, established by MyNews repository, a platform that records all the information published in the main Spanish media on the same dates, and on similar topics. Tables 1 and 2 show the 20 selected claims, 10 misinformation news and 10 legitimate news.

Table 1. Misinformation news

\begin{tabular}{|c|l|}
\hline New id & Claim \\
\hline 11 & Alimentos que más inmunizan contra el coronavirus \\
\hline 12 & Hantavirus. La OMS advierte al mundo del nuevo virus que viene de China \\
\hline 13 & $\begin{array}{l}\text { Madrid denuncia que el Gobierno paralizó en Zaragoza 5.000 kilos de mas- } \\
\text { carillas para Madrid porque «Aduanas cierra a las 15h» }\end{array}$ \\
\hline 14 & $\begin{array}{l}\text { Bill Gates anuncia que implantará microchips para combatir Covid-19 y } \\
\text { rastrear las vacunas }\end{array}$ \\
\hline 15 & $\begin{array}{l}\text { Demuestran científicamente la relación causal entre la tecnología 5G y el } \\
\text { COVID-19 }\end{array}$ \\
\hline 16 & $\begin{array}{l}\text { Todo apunta a que el COVID-19 "se escapó" del Instituto de Virología de } \\
\text { Wuhan: ¿Desarrollaban los chinos un arma de guerra biológica? }\end{array}$ \\
\hline 17 & \begin{tabular}{l} 
El uso prolongado del tapaboca produce hipoxia \\
\hline 18
\end{tabular} Stefano Montario: las mascarillas incuban el cáncer \\
\hline 19 & $\begin{array}{l}\text { El Ministerio del Interior alemán define al coronavirus como «falsa alarma } \\
\text { global» en un informe filtrado a la prensa }\end{array}$ \\
\hline 20 & La OMS alerta sobre el Virus Nipah que puede ser peor que el Covid-19 \\
\hline
\end{tabular}

\footnotetext{
4 This project is financed by the BBVA Foundation in its 2019 call for Ayudas a Equipos de Investigación Científica en el área de Economía y Sociedad Digital. https://www.rrssalud.org

${ }^{5}$ Sources: EFE Verifica, Maldita and Newtral
} 
Table 2. Legitimate news

\begin{tabular}{|c|l|}
\hline New id & Claim \\
\hline 1 & $\begin{array}{l}\text { La Junta iniciará el lunes el reparto de menús para menores en riesgo de } \\
\text { exclusión en Córdoba }\end{array}$ \\
\hline 2 & $\begin{array}{l}\text { El CSIC busca una vacuna contra el Covid-19 a partir del virus que erradicó } \\
\text { la viruela }\end{array}$ \\
\hline 3 & $\begin{array}{l}\text { Coronavirus: llega a Madrid el material sanitario al que Huawei se compro- } \\
\text { metió con el rey Felipe VI }\end{array}$ \\
\hline 4 & No hay evidencia de que los perros transmitan el virus \\
\hline 5 & $\begin{array}{l}\text { Moscú aprovecha el coronavirus para imponer el control a la población con } \\
\text { códigos QR }\end{array}$ \\
\hline 6 & El coronavirus solo se desactiva por completo a más de 90 grados \\
\hline 7 & El uso de la mascarilla agrava el aislamiento de las personas sordas \\
\hline 8 & Utilizar guantes no tiene ningún sentido \\
\hline 9 & Evitar el coronavirus con la vacuna de la polio \\
\hline 10 & Sanidad estudia adelantar este año la vacuna de la gripe \\
\hline
\end{tabular}

In addition, we have collected tweets using the official Twitter API related to the news from our dataset. For each of them, we searched a first set of tweets containing the URL of these news or their claim, starting on March 2020 until May 2021. From this initial set of $2.5 \mathrm{k}$ tweets we then captured the whole conversation around those tweets, the retweets and the quotes and collect a total of $20.5 \mathrm{k}$ tweets. Finally we collected the users set composed of the tweets' authors and the users who liked or retweeted any tweet from the initial set $(\mathrm{N}=40.1 \mathrm{k})$.

With this dataset we built the tweet network $\mathrm{T}$ and user network $\mathrm{U}$, as well as the resulting two one-mode projection of the bipartite network B, where the nodes $N_{t} \cup N_{u} . N_{t}$ are the nodes representing the tweets and $N_{u}$ are the nodes representing users. An edge $e_{t u}$ represents the interaction (like or retweet) from the user $u$ to the tweet $t$. Since we are interested in the influence and spreading of the news, we based our graph on retweets and likes. This is an important aspect to retain as all the metrics under this graph can only be interpreted based on its foundation. An edge between node $t_{1}$ and node $t_{2}$ is created if they have a common user that has interacted with both tweets. Finally, graph U represents the users network and its nodes represent users. Again, an edge between user $u_{1}$ and user $u_{2}$ is created if they interacted with a common tweet.

Since the tweets are collected from different sources of selected misinformation news, tweets that are related to a misinformation news are labelled as misinformation tweets, and tweets that are related to a legitimate new are labelled as legitimate tweets.

\section{Tweets analysis}

\subsection{Graphs analysis}

In terms of the type of news gathered, our graph $\mathrm{T}$ presents an unbalance in favour for the misinformation tweets as depicted in Figure 1 . We have identified 
16601 tweets related to the misinformation news, while only 3905 are about legitimate news. Thus, misinformation news were tweeted four times more than legitimate news.

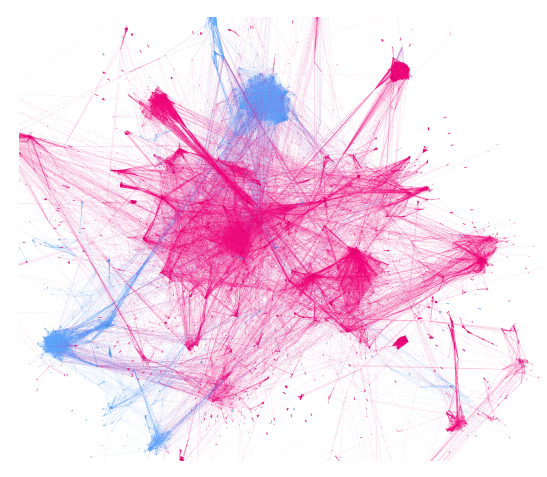

Fig. 1. Graph T with pink coloured misinformation news and blue coloured legitimate news

We also found unbalance in regards to the news proportion. As shown in Table 3 most of the tweets are about the news $\# 14$, and we have barely some tweets from news \#15 and \#12 and nothing for news \#11 and \#20.

For the legitimate news tweets, as shown in Table 4, most of the tweets also comes from a single item, the news \#3. Moreover, we did not find any tweet coming from news $\# 1, \# 2, \# 5$, and $\# 7$. Results show that legitimate news have more potential to go unnoticed, compared to misinformation news.

Table 3. Misinformation news distribution

\begin{tabular}{|r|ccc|}
\hline New id & Nodes percentage Mean degree Mean retweet influence coefficient \\
\hline 11 & $0 \%$ & - & - \\
12 & $0.63 \%$ & 5.80 & 0.37 \\
13 & $4.38 \%$ & 18.13 & 0.24 \\
14 & $54.59 \%$ & 21.31 & 0.22 \\
15 & $0.55 \%$ & 9.15 & 0.51 \\
16 & $2.03 \%$ & 4.74 & 0.30 \\
17 & $1.80 \%$ & 11.04 & 0.04 \\
18 & $8.03 \%$ & 20.00 & 0.19 \\
19 & $16.45 \%$ & 22.07 & 0.22 \\
20 & $0 \%$ & - & - \\
\hline
\end{tabular}

To find if different tweets are related by the same group of people we built the sub-graph $T_{\text {initial }}$ of the graph $\mathrm{T}$ where the nodes are only the tweets collected in the initial phase of the data collection, and discarded the nodes that are part 
Table 4. Legitimate news distribution

\begin{tabular}{|r|ccc|}
\hline New id & \multicolumn{3}{|c|}{ Nodes percentage Mean degree Mean retweet influence coefficient } \\
\hline 1 & $0 \%$ & - & - \\
2 & $0 \%$ & - & - \\
3 & $12.45 \%$ & 34.67 & 0.79 \\
4 & $0.03 \%$ & 1 & 0.88 \\
5 & $0 \%$ & - & - \\
6 & $2.19 \%$ & 17.68 & 0.79 \\
7 & $0 \%$ & - & - \\
8 & $2.19 \%$ & 14.27 & 0.76 \\
9 & $0.03 \%$ & 3.50 & 0.99 \\
10 & $0.09 \%$ & 4 & 0.93 \\
\hline
\end{tabular}

of the conversation of those tweets (see Section 2). The goal was to remove the tweets that were related to each other as they were part of the same conversation in order to analyse the connections between different and independent tweets. The resulting graph $T_{\text {initial }}$ is a graph where most of the nodes have no connection at all. However, those nodes who have connections belong only to misinformation news.

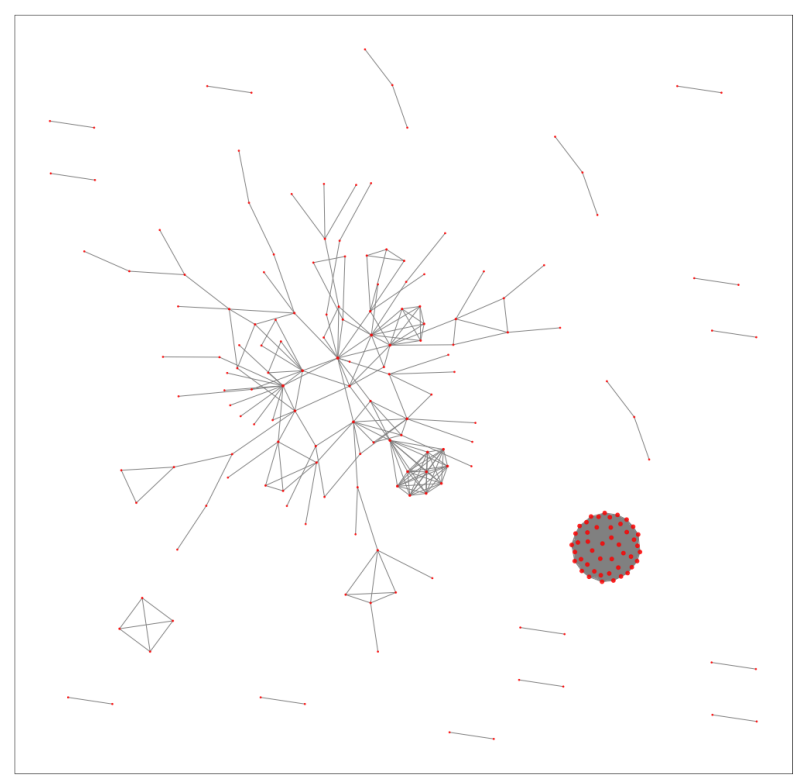

Fig. 2. Subgraph of initial tweets

The Figure 2 represents the graph of the nodes that had at least one connection from the mentioned graph. Tweets mentioning misinformation news are 
depicted with red dots, while tweets mentioning legitimate news are in blue. As observed, only red nodes are represented 6 . We interpret this as there is only a connection between the users interacting with misinformation news. Thus, a user who interacts with a tweet containing misinformation is more probable for it to interact with another tweet containing misinformation. This reinforces the findings from 77. To compare how nodes from the same type of news tend to connect to nodes from their similar type we calculated the discrete assortativity coefficient [9] for the graph $\mathrm{T}$ described in Section 2. We get a discrete assortativity of 0.898 . A high assortativity coefficient means that nodes do not tend to be connected if they are different, so there are few links between misinformation news nodes and legitimate news nodes which means there are few users that interact with both misinformation tweets and legitimate tweets. This reinforce the idea that users tend to interact with the same type of news.

\subsection{Tweets influence}

On a general perspectives, influence has been extensively studied using users on Twitter [12], as well as the measure of the tweet influence itself [4]. These metrics measure the influence of tweets by counting their number of retweets following the logic that if a tweet is retweeted it means the message in the tweet was strong enough for the user to share it with others and being presented to a new set of users, contributing to the spread of the news. Previous studies have shown that the number of retweets follows a power law distribution [5]. Hence, our following analysis aims to verify whether the number of tweet count per number of retweets also followed a power law. One way to measure the influence of a tweet could by the degree centrality measure [10. However, this method is not adequate in our case, due to the limitations for the graph construction It does not represent a global measure of influence but rather in the context of the graph, and the way it was built. Another way to measure the influence of a tweet is by counting how many times the tweets has been retweeted.

We fitted the tweet counts by number of retweets to a power law using the tool developed in [1] and observed that they follow a power law with an $\alpha=1.80$ as seen in Figure 3. This means that tweets that have a lot of retweets, and by our definition are more influential, are less frequent. We can have a coefficient of how influential a tweet is by the retweets and the Probability Density Function (PDF) of the power law.

$$
c_{i}(t)=1-r_{t}^{-\alpha}
$$

Equation 1 shows us this Retweet Influence Coefficient (RIC) $c_{i}$ for a tweet $t$ which has $r_{t}$ retweets and in our case $\alpha=1.80$. Since we want the tweets with more influence to have a higher RIC we took the inverse of the PDF by subtracting it to 1 .

\footnotetext{
6 The figure shows a big clique of 45 nodes, those tweets are the same tweets being posted by the same user and this user liking its own tweets
} 


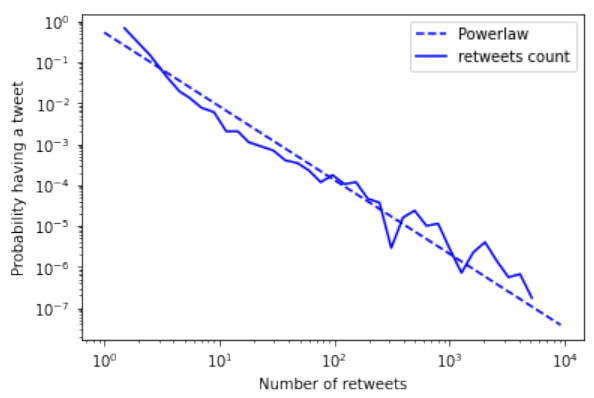

Fig. 3. Powerlaw fitting to the tweet count by number of retweets

In Table 3 we can see that the tweets that are the most connected are not necessarily the one with the most influence. One of the causes of this, might be to the graph construction.

Doing a qualitative investigation about the difference between the tweets with high degree centrality and the tweets with high RIC, we found out that tweets that have high degree centrality do not have any particularity to be the most influential. Some were comments, some were original tweets, some had lots of replies and others barely where liked.

Therefore we cannot consider the degree centrality of a tweet as reliable measure of influence for this type of graphs.

On contrary, using our measure of RIC, the tweets with the highest RIC were tweets that carried an important message and/or where published by someone well known like an important politician.

When comparing the misinformation news RIC score $(\mathrm{RIC}=0.164)$ and the legitimate news' one $(\mathrm{RIC}=0.156)$ no significant difference were found (one-way ANOVA: $\mathrm{F}(2,4751)=1.05$, p-value $=0.41)$. Legitimate news and misinformation news have the same probability to be retweeted.

\section{User analysis}

\subsection{User graph}

For the user graph the nodes are the users, and a link between two users if they interacted with the same tweet. The resulting graph contains 32500 nodes and 1006998 edges. The mean degree in the graph is 63.61 , the maximum 821 , and the minimum is 1 . Having a higher degree than in the tweets graphs is probably due to the fact that users tend to interact with the tweets in the same context and even more in the same conversation.

Table 5 shows that the most connected users are also the ones who are the most active (produce more tweets), have more followers and follow more people.

In case a user interacted or authored more tweets related to misinformation news it was marked as misinformation user, otherwise it was marked as legitimate 
Table 5. Highest degree nodes compared to the average node

\begin{tabular}{|r|ccc|} 
Measure & General Mean Mean 100 highest degree Mean 10 highest degree \\
\hline Tweets posted & 30141.27 & 119146.60 & 219308.70 \\
Followers & 1466.63 & 4427.49 & 2405.50 \\
Following & 1204.13 & 2055.07 & 2852.60
\end{tabular}

user. We found that for a total of 31629 users, 25703 users were misinformation users and 5926 were legitimate users. This is coherent with the nature of our dataset.

If we calculate the discrete assortativity coefficient $[9$ in the users graph $\mathrm{U}$ to measure how nodes connect to similar nodes when comparing about misinformation users and legitimate users, we get a 0.88 coefficient which is high. This is expected since legitimate users will interact with legitimate news and misinformation users interact with misinformation news according to our definition.

In addition, we clustered the users based on their graph U using the Louvain algorithm [3]. Then we identified those clusters where there were a mix of legitimate and misinformation users. There are 70 groups, with each group having mean size of 295.3 users. With the smallest group being of 3 users and the biggest group being of 1877 users.

Table 6. 10 biggest communities that contain misinformation and legitimate users

\begin{tabular}{|c|c|c|c|}
\hline Community & Legitimate Users & Misinformation Users & Proportion \\
\hline 62 & 469 & 8 & $98.32 \%-1.68 \%$ \\
\hline 14 & 5 & 508 & $0.97 \%-99.03 \%$ \\
\hline 49 & 106 & 557 & $15.99 \%-84.01 \%$ \\
\hline 11 & 602 & 67 & $89.99 \%-10.01 \%$ \\
\hline 47 & 1 & 709 & $0.14 \%-99.86 \%$ \\
\hline 57 & 710 & 55 & $92.81 \%-7.19 \%$ \\
\hline 12 & 6 & 813 & $0.73 \%-99.27 \%$ \\
\hline 54 & 2 & 1010 & $0.19 \%-99.81 \%$ \\
\hline 6 & 55 & 1000 & $5.21 \%-94.79 \%$ \\
\hline 10 & 23 & 1854 & $1.23 \%-98.77 \%$ \\
\hline
\end{tabular}

Table 6 shows that most of the communities are very unbalanced in favour of a type of user. Nonetheless, we created the community sub-graph of the most balanced communities to see how users from other groups interacted with each other, which is represented in Figure 4. We can observe that even by creating mixed communities sub-graphs, the communities remains homogeneous. For example in community 49, mostly composed of misinformation users, there are 2 clusters of legitimate users. In communities 11 and 57, mostly composed by legitimate users, most of the misinformation users are in the peripheries of the cluster or linked by bridge users to the legitimate users. In conclusion legitimate users tend to connect to legitimate users, and misinformation users tend to con- 


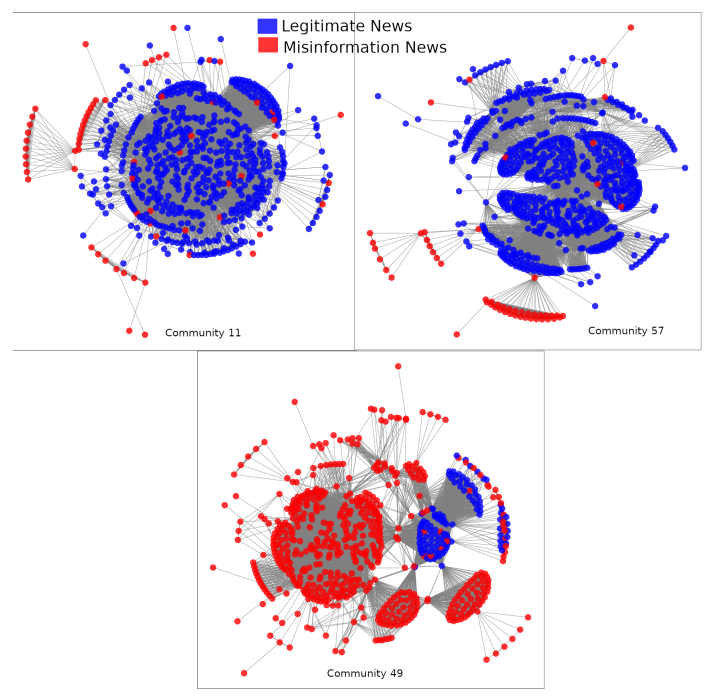

Fig. 4. Mixed user communities

nect to misinformation even in the case of mixed communities and even when connection between the two communities exist, they are only connected through a few users.

\subsection{User influence}

Our analysis to measure the influence of users in Twitter is based on a metric proposed by 11.

They defined the Retweet Impact as shown in Equation 2. Where $O G$ is the number of original tweets, and $R T$ is the number of users who have retweeted the author's tweets.

$$
R I=O G \cdot \log (R T)
$$

For our case we used the total count of retweets instead of the total of different users that have retweeted at least once one of the author's tweets to account from the Twitter's API limits of 100 users retweets data collection.

A qualitative analysis of the 100 most influential users, we found that 78 were misinformed (38 from Spain) and 22 were legitimate users (18 from Spain). Despite having 81 percent of the total users misinformed, this proportion is not maintained with the most influential users. Even though there users interacting with misinformation, those users are not necessarily more influential than legitimate users.

We also found out that most of the misinformed users from this list of 100 most influential post content about politics, and religion. Where 13 of those users post 
content related to extreme right ideas, 4 of those users post content related to extreme left ideas, and finally 3 of those users post religious content. Most of the legitimate users from the list of 100 most influential users post post content about scientific diffusion and fight against misinformation.

Finally we found that from this list of 100 most influential users, 44 users are not from Spain or their region could not be identified.

\subsection{Users location}

We developed a tool to identify the location of the users based on their location input and their description. Even though we selected news from Spanish sources we could not avoid getting users from other countries specially from Latin America. As shown in Table 7, for Spanish users, our user sample reflect the proportion of the different provinces.

Table 7. Users, authors of initial dataset, per Spanish localisation

\begin{tabular}{l|c} 
Location & Number of users \\
\hline Unknown & 2513 \\
Comunidad de Madrid & 168 \\
Cataluña & 106 \\
España & 65 \\
Andalucía & 46 \\
Galicia & 44 \\
Comunidad Valenciana & 43 \\
Castilla y León & 10 \\
Principado de Asturias & 19 \\
Canarias & 16 \\
Región de Murcia & 15 \\
Others Spanish regions & 41
\end{tabular}

A qualitative review from a sample from users that have an unknown location showed that around one third of those users still are from Spain. The rest of the users where in minority from an unknown location or from a Spanish speaking country. Nevertheless we cannot ignore the rest of the users coming from another country. We could say that the level of misinformation also depends from the level of misinformation in other Spanish speaking countries.

\section{Discussion and future work}

In this study we analysed the tweet influence among conversations on Twitter about misinformation and legitimate news. Our goal was to develop a better understanding of the different news spread patterns, as well as tackling some limitations inherent to influence metrics. A common issue raised by metrics, such as degree centrality measure, its their dependency upon the graph construction 
process itself. This led us to develop the RIC measure which has the advantages to be independent to the graph structure and capturing the influence of the tweets appropriately.

While being more adequate to unveil influential tweets, our influence metric did not show any significant difference between misinformation and verified news, arguing for a similar impact of both type of news reveal a difference in the users behaviour. Users that spread and/or interact with misinformation tend to post more about it than users who post about verified news. This effect was also found in [2], where authors observed that misinformation users are more focused on spreading their ideas than legitimate users.

But since there is not a significant difference in the influence of misinformation tweets compared to legitimate tweets, both news are shared the same amount of times. Misinformation is being posted more often, resulting in a wider spread thus a different pattern of communication where users exhibit different behaviours if they tend to post about misinformation rather than legitimate news. No only does the users from different groups behave differently, they also do not interact with each others. They tend to interact with other users that share the same type of news than them and thus are prone to the echo chamber effect, confirming finding from previous studies [7] [2].

Another important aspect in term of combating misinformation is the language. Users are connected with people from other countries that speak the same language. This raises the concern of how do spread patterns of misinformation behave. Our findings indicate that language prevails over geographic factors, exacerbating the difficulties for governments to control the dissemination of misleading information.

\section{References}

1. Alstott, J., Bullmore, E., Plenz, D.: Powerlaw: a Python package for analysis of heavy-tailed distributions. PLoS ONE 9(1), e85777 (Jan 2014). https://doi.org/10.1371/journal.pone.0085777, http://arxiv.org/abs/1305. 0215 arXiv: 1305.0215

2. Bessi, A., Coletto, M., Davidescu, G.A., Scala, A., Caldarelli, G., Quattrociocchi, W.: Science vs Conspiracy: Collective Narratives in the Age of Misinformation. PLOS ONE 10(2), e0118093 (Feb 2015). https://doi.org/10.1371/journal.pone.0118093 https://journals.plos.org/ plosone/article?id=10.1371/journal.pone.0118093, publisher: Public Library of Science

3. Blondel, V.D., Guillaume, J.L., Lambiotte, R., Lefebvre, E.: Fast unfolding of communities in large networks. Journal of Statistical Mechanics: Theory and Experiment 2008(10), P10008 (Oct 2008). https://doi.org/10.1088/17425468/2008/10/P10008, http://arxiv.org/abs/0803.0476 arXiv: 0803.0476

4. Huang, B., Carley, K.M.: Disinformation and Misinformation on Twitter during the Novel Coronavirus Outbreak. arXiv:2006.04278 [cs] (Jun 2020), http://arxiv. org/abs/2006.04278, arXiv: 2006.04278

5. Mathews, P., Mitchell, L., Nguyen, G., Bean, N.: The Nature and Origin of Heavy Tails in Retweet Activity. In: Proceedings of the 26th International Conference 
on World Wide Web Companion - WWW '17 Companion. pp. 1493-1498. ACM Press, Perth, Australia (2017). https://doi.org/10.1145/3041021.3053903, http: //dl.acm.org/citation. cfm?doid=3041021.3053903

6. McQuillan, L., McAweeney, E., Bargar, A., Ruch, A.: Cultural Convergence: Insights into the behavior of misinformation networks on Twitter. arXiv:2007.03443 [physics] (Jul 2020), http://arxiv.org/abs/2007.03443, arXiv: 2007.03443

7. Memon, S.A., Carley, K.M.: Characterizing COVID-19 Misinformation Communities Using a Novel Twitter Dataset. arXiv:2008.00791 [cs] (Sep 2020), http: //arxiv.org/abs/2008.00791, arXiv: 2008.00791

8. Myers, S.A., Sharma, A., Gupta, P., Lin, J.: Information network or social network?: the structure of the twitter follow graph. In: Proceedings of the 23rd International Conference on World Wide Web. pp. 493-498. ACM, Seoul Korea (Apr 2014).https://doi.org/10.1145/2567948.2576939, https://dl .acm.org/doi/ $10.1145 / 2567948.2576939$

9. Newman, M.E.J.: Mixing patterns in networks. Physical Review E 67(2), 026126 (Feb 2003). https://doi.org/10.1103/PhysRevE.67.026126, http://arxiv . org/abs/cond-mat/0209450, arXiv: cond-mat/0209450

10. Nieminen, J.: On the centrality in a graph. Scandinavian Journal of Psychology 15(1), 332-336 (1974). https://doi.org/10.1111/j.1467-9450.1974.tb00598.x, https://onlinelibrary.wiley.com/doi/abs/10.1111/j.1467-9450.1974. tb00598.x

11. Pal, A., Counts, S.: Identifying topical authorities in microblogs. In: Proceedings of the fourth ACM international conference on Web search and data mining - WSDM '11. p. 45. ACM Press, Hong Kong, China (2011). https://doi.org/10.1145/1935826.1935843 http://portal.acm.org/citation. cfm?doid=1935826.1935843

12. Riquelme, F., González-Cantergiani, P.: Measuring user influence on Twitter: A survey. Information Processing \& Management 52(5), 949-975 (Sep 2016). https://doi.org/10.1016/j.ipm.2016.04.003 http://arxiv.org/abs/1508. 07951, arXiv: 1508.07951

13. Salaverría, R., Buslón, N., López-Pan, F., León, B., López-Goñi, I., Erviti, M.C.: Desinformación en tiempos de pandemia: tipología de los bulos sobre la Covid-19. El Profesional de la Información 29(3) (May 2020). https://doi.org/10.3145/epi.2020.may.15 https://revista. profesionaldelainformacion.com/index.php/EPI/article/view/epi.2020. may.15

14. Singh, L., Bansal, S., Bode, L., Budak, C., Chi, G., Kawintiranon, K., Padden, C., Vanarsdall, R., Vraga, E., Wang, Y.: A first look at COVID-19 information and misinformation sharing on Twitter. arXiv:2003.13907 [cs] (Mar 2020), http: //arxiv.org/abs/2003.13907, arXiv: 2003.13907

15. Viviani, M., Pasi, G.: Credibility in social media: opinions, news, and health information - a survey. WIREs Data Mining Knowl Discov 7(e01209), 1-25 (2017). https://doi.org/10.1002/widm.1209, https://onlinelibrary.wiley.com/ doi/abs/10.1002/widm.1209 